\title{
Comparison of various methods for analyzing lined circular ducts
}

\author{
S.N. Panigrahi, M.L. Munjal* \\ Facility for Research in Technical Acoustics, Department of Mechanical Engineering, Indian Institute of Science, \\ Bangalore 560012, India
}

\begin{abstract}
Over the years, lined ducts with bulk reacting linings have been investigated for their sound attenuation performance. The methods employed for these studies can be broadly categorized into three major types. In one approach, the duct is assumed to be of infinite length of which a slice of finite length is taken out to get a transfer matrix between the two end points of this slice. In another approach, the one dimensional, coupled wave equations are solved, with the pressure difference between the flow passage and the absorptive lining being supported by a perforated plate. In the third major approach, the three-dimensional wave equation is solved, taking into consideration the finite length boundary effects. In this paper, these three approaches are compared with respect to their limitations, simplicity and accuracy. Here, the lowest mode or the $(0,0)$ mode only has been investigated. In the third approach, improvements are incorporated to make this method work accurately in the high frequency regions too. Relatively larger sized ducts are also examined for different values of $d / h$ ratios.
\end{abstract}

\section{Introduction}

Dissipation of acoustical energy in absorptive linings is the most widely analyzed sound attenuation concept in the gas flow containing absorptive ducts, where minimization of pressure

\footnotetext{
${ }^{*}$ Corresponding author. Tel. : + 91802293 2303; fax: + 918023600648.

E-mail address: munjal@mecheng.iisc.ernet.in (M.L. Munjal).
} 


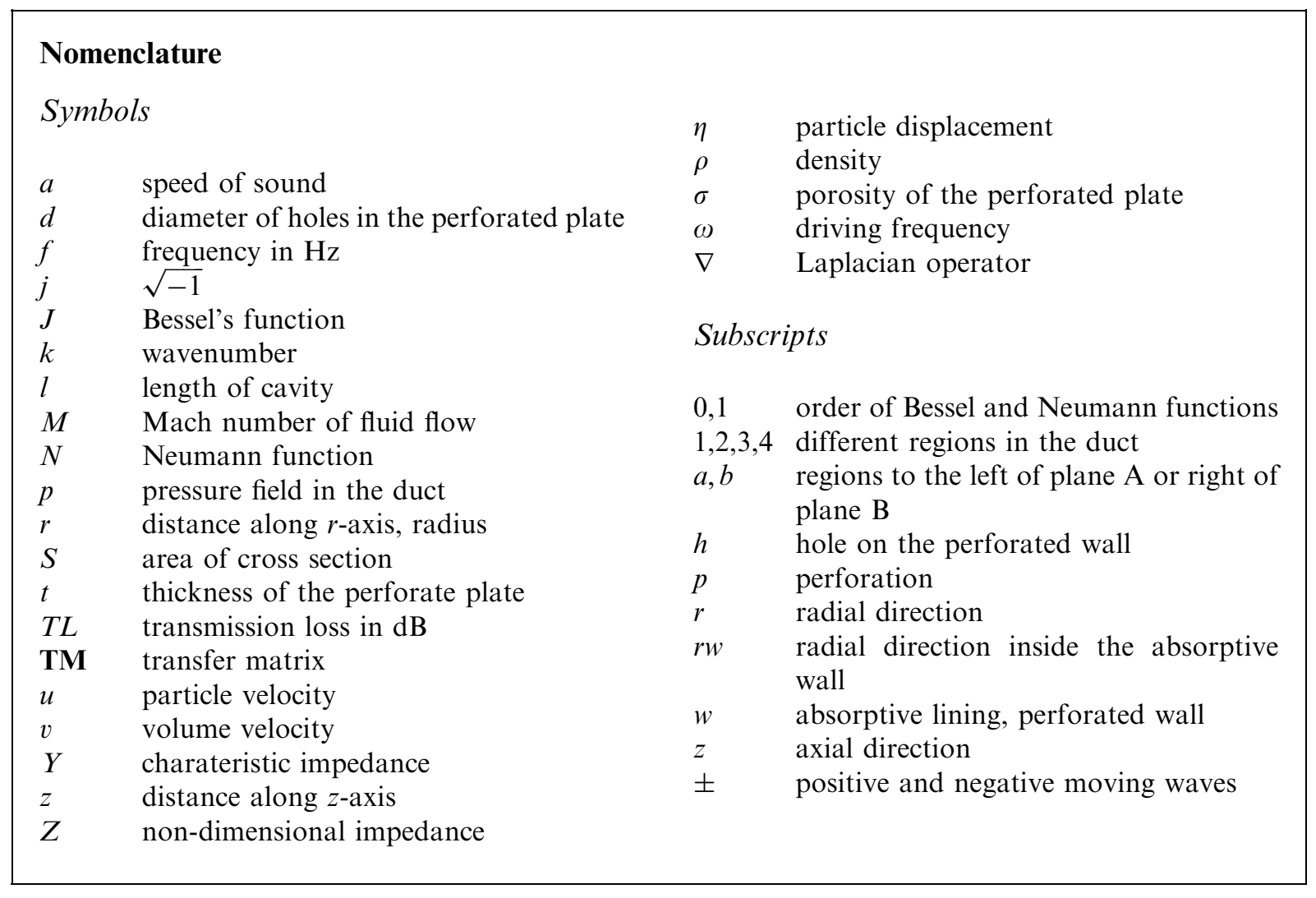

drop across the silencer is also of utmost concern with the primary objective of broadband sound attenuation. Their frequent use in the intake and exhaust ducts of gas turbines, air conditioning and ventilation ducts of large industrial fans, etc. necessitates the development of a standardized procedure to analyze these ducts under different operating conditions for the desired accuracy and the frequency range of interest.

Some situations with high volume flow rates dictate the size of the silencer to be of the order of one meter diameter or even more with very low velocity of mean flow of the exhaust or ventilated gas through these ducts. In those cases, the analysis should be stable throughout the frequency range of interest. Then, depending on the application, two kinds of configuration are generally implemented to optimize the cost of fabrication: rectangular and circular. In this paper, the circular ducts are investigated. The three methods used for getting the transfer matrix are described below. The finite-length slice approach (hereafter, referred to as the first approach or the finite-slice model) and the one-dimensional decoupling approach (referred to here as the second approach or the two-duct model, following Ref. [1]) are explained very briefly, as they follow the same arguments as in Refs. [1,2], respectively, with very little modifications. The third approach, viz. the two-dimensional wave model with finite boundary effect approach (referred to here as two-dimensional model or the third approach) is described in detail incorporating many changes to be able to investigate high frequency ranges and large sized silencers. 
It may be noted that the first approach ignores wave reflections from the end plates of the annular cavity. The second approach implies coupled one-dimensional analysis. The third approach is same as the first approach as far as the derivation of the axial wavenumber is concerned. But, for deriving the transfer matrix, the reflections at the boundaries are taken into account.

\section{First approach: a slice of finite length taken from an infinite duct}

In analyzing the bulk-reacting liner on circular ducts with a perforated protective layer, shown in Fig. 1, it can be noted that the protective layer thickness at $r=r_{1}$ is too small to be considered while calculating the lining thickness using $r_{1}$ and $r_{2}$. Then, for the fundamental or the $(0,0)$ mode forward progressive wave in the central flow duct, the pressure field can be expressed as

$$
p(z, r, t)=A J_{0}\left(k_{r} r\right) \mathrm{e}^{\mathrm{j}\left(\omega t-k_{z} z\right)},
$$

with the over-riding compatibility condition

$$
k_{z}^{2}+k_{r}^{2}=k_{0}^{2},
$$

where $J_{0}$ is the Bessel function of zeroth order. $k_{z}$ and $k_{r}$ are the wavenumbers in the axial and the radial direction. $k_{0}$ is the wavenumber in the air medium and $\omega$ is the driving frequency.

Similarly, the pressure field in the lining is given by the expression

$$
p_{w}(z, r, t)=B\left\{J_{0}\left(k_{r w} r\right)+C_{b} N_{0}\left(k_{r w} r\right)\right\} \mathrm{e}^{\mathrm{j}\left(\omega t-k_{z} z\right)},
$$

with the over-riding compatibility condition

$$
k_{z}^{2}+k_{r w}^{2}=k_{w}^{2},
$$

where $N_{0}$ is the Neumann function of zeroth order. $k_{w}$ is the wavenumber in the absorptive medium. $k_{r w}$ is the wavenumber in the radial direction inside the absorptive wall.

In writing Eq. (4) it has been assumed (inherent in the bulk reaction model) that the axial wavenumber in the lining equals that in the central duct $\left(r<r_{1}\right)$. The radial particle velocities in

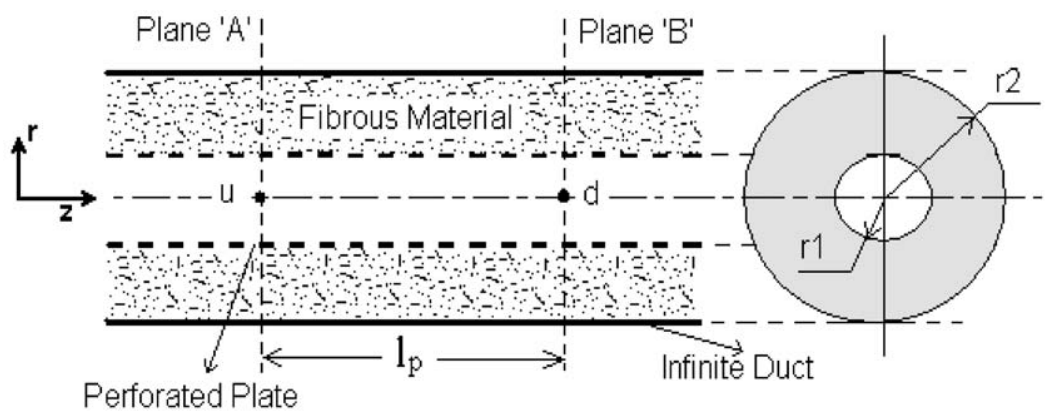

Fig. 1. Slice of an infinite circular duct lined with a bulk-reacting lining. 
both regions can be derived using the Euler's (momentum) equation as follows:

$$
\begin{gathered}
u_{r}(z, r, t)=-\mathrm{j} \frac{k_{r}}{\omega \rho_{0}} A J_{1}\left(k_{r} r\right) \mathrm{e}^{\mathrm{j}\left(\omega t-k_{z} z\right)}, \\
u_{r w}(z, r, t)=-\mathrm{j} \frac{k_{r w}}{\omega \rho_{w}} B\left[J_{1}\left(k_{r w} r\right)+C_{b} N_{1}\left(k_{r w} r\right) \mathrm{e}^{\mathrm{j}\left(\omega t-k_{z} z\right)}\right],
\end{gathered}
$$

where $\rho_{0}$ and $\rho_{w}$ are the densities of the air and the absorptive medium. $J_{1}$ and $N_{1}$ are the Bessel and Neumann functions of first order. The value of the constant $C_{b}$ can be evaluated from the zero-radial-velocity boundary condition at the outer rigid wall as

$$
C_{b}=-\frac{J_{1}\left(k_{r w} r_{2}\right)}{N_{1}\left(k_{r w} r_{2}\right)}
$$

Munjal and Thawani [3], in applying the perforate impedance formula, have used the expression for a perforated plate backed by an air cavity, on the assumption that it would hold for the lined duct as well on account of very high values of porosity of the fibrous backing. In this study, the expression for the same has been modified in the line of Ref. [2] and is discussed in detail later under the second approach.

Neglecting the convective effect of mean flow, radial velocities across the perforated layer can be equated as

$$
u_{r}\left(r_{1}\right)=u_{r w}\left(r_{1}\right)
$$

If the perforated plate is assumed to sustain a pressure difference, then the pressure drop across the perforate layer can be related to the radial particle velocity at that point as

$$
p\left(r_{1}\right)=p_{w}\left(r_{1}\right)+Z_{p} \rho_{0} a_{0} u_{r}\left(r_{1}\right),
$$

where $Z_{p}$ is the non-dimensional perforate impedance of the protective layer, discussed in detail later under the second approach and $a_{0}$ is the speed of sound in the central duct. Then, by dividing Eq. (9) by Eq. (8), and making use of Eqs. (1), (3), (5) and (6), the eigenequation can be derived as [3]

$$
\mathrm{j} \frac{\omega \rho_{0}}{k_{r}} \frac{J_{0}\left(k_{r} r_{1}\right)}{J_{0}\left(k_{r} r_{1}\right)}=\mathrm{j} \frac{\omega \rho_{w}}{k_{r w}} \frac{J_{0}\left(k_{r} r_{1}\right)+C_{b} N_{0}\left(k_{r w} r_{1}\right)}{J_{1}\left(k_{r} r_{1}\right)+C_{b} N_{1}\left(k_{r w} r_{1}\right)}+Z_{p} \rho_{0} a_{0} .
$$

After getting the eigenequation relating the axial wavenumber (both $k_{r w}$ and $k_{r}$ are functions of $k_{z}$ ) and other physical parameters of the muffler, it is solved to get the complex axial wavenumber using the Newton-Raphson scheme. As opposed to the cited reference, the initial guess for the solution scheme at the lowest frequency $(1 \mathrm{~Hz})$ is derived from a second degree polynomial approximation of the actual eigenequation [4,5]. For subsequent frequency steps, the root of the previous step is taken as the initial guess. To avoid the problem of mode jumping [6,7], a frequency step of $1 \mathrm{~Hz}$ is chosen which can still be made smaller if some mode jumping is observed for some particular configuration, as the computation time taken for each step is very little. The function derivative at each step is calculated by numerical differentiation methods [8].

Having got the complex axial wavenumber for stationary medium, the same can be calculated for the convective medium as described in Ref. [1]. Finally, the transfer matrix (or the four-pole parameters) can also be derived from which the transmission loss is readily calculated from the 
expression [1]

$$
T L=20 \log _{10}\left|\frac{\left(T_{11}+T_{12} / Y_{1}+Y_{1} T_{21}+T_{22}\right)}{2}\right|,
$$

where $T_{i j}(i, j=1,2)$ are the four-pole parameters and $Y_{1}$ is the characteristic impedance of the central duct.

\section{Second approach: two-duct model or the one-dimensional decoupling approach}

This analysis goes in the line of Ref. [1], where the transfer matrix for a concentric tube resonator has been derived using the one-dimensional decoupling approach. Certain changes are incorporated in the expression for the perforate impedance of the protective layer in the case of a grazing flow and in the boundary conditions. In the absence of mean flow, for perforations facing the air medium, the perforate impedance is given by [1]

$$
Z_{p}=\left[0.006+\mathrm{j} k_{0}\left(t_{w}+0.75 d_{h}\right)\right] / \sigma,
$$

where $t_{w}$ and $d_{h}$ are the thicknesses of the perforated wall and the diameter of the holes or perforations, respectively. $\sigma$ denotes the overall porosity of the perforated protective plate. In their paper, Selamet et al. [2] have modified the perforate impedance expression for the situation, when the materials on the two sides of the protective layer are different (refer Fig. 2).

In this case, the above impedance expression gets modified as

$$
Z_{p}=\left[0.006+\mathrm{j} k_{0}\left\{t_{w}+0.375 d_{h}\left(1+\frac{Y_{w}}{Y_{0}} \frac{k_{w}}{k_{0}}\right)\right\}\right] / \sigma .
$$

With the above expression for the perforate impedance in the one-dimensional decoupled analysis, they have demonstrated good agreement with the experimental and BEM results. But, their expressions are valid only for a stationary medium. Comparing Eq. (12) and (13), one observes that the reactive part of the perforate impedance, which is a function of the perforate-hole diameter, is getting modified by the presence of the absorptive lining in place of air on one side of the perforated plate. So, following a heuristic approach, the corresponding expression for the same in the case of a moving medium under the grazing flow assumption [1] can be expressed as

$$
\begin{aligned}
Z_{p}= & \frac{1}{\sigma}\left[7.337 \times 10^{-3}(1+72.23 M)+\mathrm{j} 2.2245 \times 10^{-5}\left\{1+51 t_{w}\right\}\right. \\
& \left.\times\left\{1+102 d_{h}\left(1+\frac{Y_{w}}{Y_{0}} \frac{k_{w}}{k_{0}}\right)\right\} f\right],
\end{aligned}
$$

where $M$ is the Mach number in the central duct and $f$ is the frequency in $\mathrm{Hz}$.

At planes ' $\mathrm{A}$ ' and ' $\mathrm{B}$ ', the boundary conditions [1] are modified to take into account the complex characteristic properties of the fibrous material as opposed to the real wavenumber and the characteristic impedance of air, that would apply if the annular region were filled with air instead of the absorptive material. 


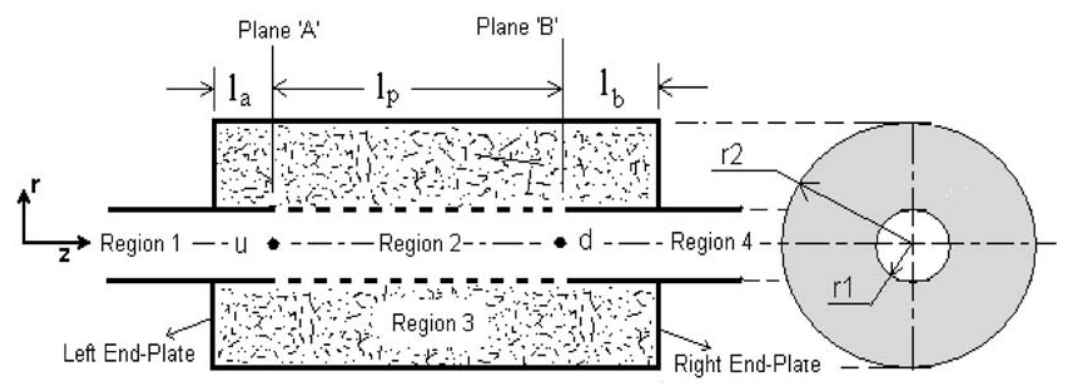

Fig. 2. The lined circular duct with perforated protective layer and extended inlet/outlet.

\section{Third approach: two-dimensional wave modeling with finite boundary effects}

To get the final transfer matrix between the upstream point ' $u$ ' and downstream point ' $d$ ' (as shown in Fig. 2), the first thing is to get the eigenequation from which the complex axial wavenumber can be got. Then, using the force equilibrium and the continuity of volume velocity at planes ' $A$ ' and ' $B$ ' in an integral sense, the finite length boundary conditions can be incorporated. These are described below under different sub-sections.

\subsection{Eigenequation formulation for a circular dissipative duct}

Waves in regions 1, 2 and 4 are governed by two-dimensional convective wave equations. Thus, for region 2 of Fig. 2

$$
\frac{1}{a_{0}^{2}} \frac{D^{2} p_{2}}{D t^{2}}-\nabla^{2} p_{2}=0
$$

Assuming sinusoidal variation w.r.t. time, Eq. (15) can be re-written as a convective Helmholtz equation:

$$
\left(1-M^{2}\right) \frac{\partial^{2} p_{2}}{\partial z^{2}}+\frac{1}{r} \frac{\partial p_{2}}{\partial r}+\frac{\partial^{2} p_{2}}{\partial r^{2}}+k_{0}^{2} p_{2}-2 \mathrm{j} M k_{0} \frac{\partial p_{2}}{\partial z}=0 .
$$

The corresponding Helmholtz equation in region 3, for stationary medium, can now be written as

$$
\frac{\partial^{2} p_{3}}{\partial z^{2}}+\frac{1}{r} \frac{\partial p_{3}}{\partial r}+\frac{\partial^{2} p_{3}}{\partial r^{2}}+k_{w}^{2} p_{3}=0
$$

where $k_{w}$ is the (complex) wavenumber of the absorptive material.

As explained under the first approach, the acoustic fields in regions 2 and 3 may be expressed by Eqs. (1) and (3) with slight modification to take into account the convective effect of the mean flow. Then, for the forward and rearward moving progressive waves, respectively, the pressure fields are given by [1]

$$
\begin{gathered}
p(z, r, t)=A_{ \pm} J_{0}\left(k_{r_{ \pm}} r\right) \mathrm{e}^{\mathrm{j}\left(\omega t-k_{z_{ \pm}} z\right)} \\
p_{w}(z, r, t)=B_{ \pm}\left\{J_{0}\left(k_{r w_{ \pm}} r\right)+C_{b_{ \pm}} N_{0}\left(k_{r w_{ \pm}} r\right)\right\} \mathrm{e}^{\mathrm{j}\left(\omega t-k_{z_{ \pm}} z\right)} .
\end{gathered}
$$


These pressure fields cannot sustain themselves independently as these regions are physically connected. So, the pressure amplitudes, $A_{ \pm}$and $B_{ \pm}$, are related to each other, respectively, so as to establish a coupled pressure field in the chamber. Now, this coupled sound field in the annular cavity region (between planes A and B, see Fig. 2) may be expressed as

$$
p_{p}(z, r)=P_{p_{+}} \psi_{p_{+}}(r) \mathrm{e}^{-\mathrm{j} k_{z_{+}} z}+P_{p_{-}} \psi_{p_{-}}(r) \mathrm{e}^{+\mathrm{j} k_{z-} z},
$$

where $\psi_{p_{ \pm}}$represents the radial dependence of the pressure field, which can be written in a divided form for the two regions as

$$
\psi_{p_{ \pm}}=\left\{\begin{array}{ll}
\psi_{2 p_{ \pm}}, & 0<r<r_{1} \\
\psi_{3 p_{ \pm}}, & r_{1}<r<r_{2}
\end{array}\right\},
$$

where $\psi_{2 p_{ \pm}}$and $\psi_{3 p_{ \pm}}$are the modal eigenfunctions prevailing in regions 2 and 3 to make the pressure amplitudes in these regions dependent on each other. In fact, $\psi_{2 p_{ \pm}}$are the ratios of the respective pressure amplitudes in the two regions at $r=r_{1}$, so as to maintain a proper pressure difference at the junction of the two regions to allow the continuity of the radial particle displacement, denoted by $\eta$, following Ref. [1], at this boundary. The pressure drop and the continuity of the radial particle displacement at the boundary can be written as [3]

$$
p\left(r_{1}\right)-p_{w}\left(r_{1}\right)=Z_{p} \rho_{0} a_{0} u_{r}\left(r_{1}\right),
$$

where $u_{r}=\mathrm{j} \omega \eta_{r}$, and

$$
\eta_{r}\left(r_{1}\right)=\eta_{r w}\left(r_{1}\right),
$$

where $Z_{p}$ is the non-dimensional perforate impedance of the protective layer (discussed in detail under the second approach). Terms $\eta_{r}(r)$ and $\eta_{r w}(r)$ are the radial particle displacements in regions 2 and 3 , respectively. Now, $\eta_{r}$ and $\eta_{r w}$ are expressed as

$$
\eta_{r}=\frac{1}{\rho_{0} \omega^{2}\left(1 \mp M\left(k_{z}^{ \pm} / k_{0}\right)\right)} \frac{\partial p}{\partial r}
$$

and

$$
\eta_{r w}=\frac{1}{\rho_{w} \omega^{2}} \frac{\partial p_{w}}{\partial r} .
$$

Making use of Eq. (22) with Eqs. (18) and (19), the ratio $A_{ \pm} / B_{ \pm}$can be got, which is nothing but the modal eigen function $\psi_{2 p_{ \pm}}$. So the eigenfunctions can now be written as

$$
\psi_{2 p_{ \pm}}=J_{0}\left(k_{r_{ \pm}} r\right) \frac{J_{0}\left(k_{r w_{ \pm}} r_{1}\right)-C_{b_{ \pm}} N_{0}\left(k_{r w_{ \pm}} r_{1}\right)}{J_{0}\left(k_{r w_{ \pm}} r_{1}\right)+\mathrm{j} \frac{z_{p} k_{r_{ \pm}} k_{0} J_{1}\left(k_{r_{ \pm}} r_{1}\right)}{\left(k_{0} \mp M k_{z \pm}\right)^{2}}}
$$

and

$$
\psi_{3 p_{ \pm}}=J_{0}\left(k_{r w_{ \pm}} r_{1}\right)-C_{b_{ \pm}} N_{0}\left(k_{r w_{ \pm}} r_{1}\right)
$$


and the term $C_{b_{ \pm}}$in the above equations can directly be written in a form analogous to Eq. (7) by replacing $k_{r w}$ with $k_{r w_{ \pm}}$to become

$$
C_{b_{ \pm}}=-\frac{J_{1}\left(k_{r w_{ \pm}} r_{2}\right)}{N_{1}\left(k_{r w_{ \pm}} r_{2}\right)} .
$$

Using Eqs. (24) and (25) in the displacement continuity Eq. (23), one can get another relation between $A_{ \pm}$and $B_{ \pm}$. So, on eliminating these constant terms, the eigenequation can be derived to be

$$
\begin{gathered}
\left(\frac{k_{r w}}{k_{r}}\right)\left[\frac{\left(1 \mp M\left(k_{z \pm} / k_{0}\right)\right)^{2} J_{0}\left(k_{r_{ \pm}} r_{1}\right)}{J_{1}\left(k_{r_{ \pm}} r_{1}\right)}+\mathrm{j} \frac{Z_{p} k_{r}}{k_{0}}\right] \\
-\left(\frac{\rho_{r w}}{\rho_{r}}\right) \frac{\left[J_{0}\left(k_{r w_{ \pm}} r_{1}\right)+C_{b_{ \pm}} N_{0}\left(k_{r w_{ \pm}} r_{1}\right)\right]}{\left[J_{1}\left(k_{r w_{ \pm}} r_{1}\right)+C_{b_{ \pm}} N_{1}\left(k_{r w_{ \pm}} r_{1}\right)\right]}=0 .
\end{gathered}
$$

This equation reduces to the eigenequation derived in the case of first approach for the no-flow condition, i.e. for $M=0$. This equation is solved for the complex axial wavenumber using the Newton-Raphson scheme. The details of the solving procedure has been discussed under the first approach. The non-convective eigen equation is solved first, and the wavenumber is then modified to take into account the convective effect and finally get $k_{z_{ \pm}}$. However, derivation of the eigenfunctions $\psi_{p_{ \pm}}$incorporates the convective effects, which is also desirable, as they are used to implement the boundary conditions in the case of sound propagation in a moving medium.

\subsection{Derivation of the four-pole parameters}

The assumption of plane wave propagation in regions 1 and 4 allows the sound pressure fields in these regions to be expressed as

$$
\begin{gathered}
p_{1}(z, r)=P_{1_{+}} \mathrm{e}^{-\mathrm{j} k_{1 z_{+}} z}+P_{1_{-}} \mathrm{e}^{+\mathrm{j} k_{1 z_{-}} z}, \\
p_{4}\left(z^{\prime}, r\right)=P_{4_{+}} \mathrm{e}^{-\mathrm{j} k_{4 z_{+}} z^{\prime}},
\end{gathered}
$$

$z^{\prime}$ is measured from the starting of region 4, that is, point ' $\mathrm{d}$ '. It can be noted that the end condition may be assumed to be anechoic, without loss of generality for derivation of the fourpole parameters, making $P_{4_{-}}=0$. The force balance equation at plane $\mathrm{A}$ is given by

$$
S_{1}\left(P_{1_{+}}+P_{1_{-}}\right)=P_{p_{+}} S_{2_{+}}+P_{p_{-}} S_{2_{-}},
$$

The force balance equation at plane $\mathrm{B}$ is given by

$$
S_{4} P_{4_{+}}=P_{p_{+}} S_{2_{+}} \mathrm{e}^{-\mathrm{j} k_{z_{+}} l_{p}}+P_{p_{-}} S_{2_{-}} \mathrm{e}^{+\mathrm{j} k_{z_{-}} l_{p}} .
$$

In the above equations,

$$
S_{1}=\pi r_{1}^{2}, \quad S_{4}=\pi r_{4}^{2}
$$

and

$$
S_{2_{+}}=2 \pi \int_{0}^{r_{1}} \psi_{2 p_{+}}(r) r \mathrm{~d} r, \quad S_{2_{-}}=2 \pi \int_{0}^{r_{1}} \psi_{2 p_{-}}(r) r \mathrm{~d} r .
$$


Eqs. (32) and (33) can now be re-written as

$$
p_{1}=P_{p_{+}}\left(\frac{S_{2_{+}}}{S_{1}}\right)+P_{p_{-}}\left(\frac{S_{2_{-}}}{S_{1}}\right)
$$

and

$$
p_{4}=P_{p_{+}}\left(\frac{S_{2_{+}}}{S_{4}}\right) \mathrm{e}^{-\mathrm{j} k_{z_{+}} l_{p}}+P_{p_{-}}\left(\frac{S_{2_{-}}}{S_{4}}\right) \mathrm{e}^{+\mathrm{j} k_{z-} l_{p}} .
$$

Now, coming to the particle velocity, from the Euler's equations in the central air-way, for the forward progressive wave, one has

$$
\rho_{0} \frac{D u_{2_{+}}}{D t}=-\frac{\partial p}{\partial z}
$$

or

$$
\mathrm{j} \omega \rho_{0}\left(1-\frac{U}{\omega} k_{z_{+}}\right) u_{2_{+}}=P_{p_{+}} \psi_{2 p_{+}}\left(\mathrm{j} k_{z_{+}}\right),
$$

where $U$ is the velocity of the fluid flow. Then one can write

$$
u_{2_{+}}=P_{p_{+}} \psi_{2 p_{+}} \frac{k_{z_{+}} / k_{0}}{\rho_{0} c_{0}\left(1-M\left(k_{z_{+}} / k_{0}\right)\right)} .
$$

So, for the forward and rearward waves in the central air-way, respectively, one has,

$$
u_{2_{ \pm}}=P_{p_{ \pm}} \psi_{2 p_{ \pm}} \alpha_{2_{ \pm}}
$$

where

$$
\alpha_{2_{ \pm}}=\frac{k_{z^{ \pm}} / k_{0}}{\rho_{0} c_{0}\left(1 \mp M\left(k_{z^{ \pm}} / k_{0}\right)\right)} .
$$

Similarly, in the annular absorptive region, assuming no convective effect in that region, for the forward and the rearward waves, respectively, one gets,

$$
u_{3_{ \pm}}=P_{p_{ \pm}} \psi_{3 p_{ \pm}} \alpha_{3_{ \pm}}
$$

where

$$
\alpha_{3_{ \pm}}=\left(\frac{k_{z^{ \pm}} / k_{0}}{\rho_{0} c_{0}}\right)\left(\frac{\rho_{0}}{\rho_{w}}\right) .
$$

Now, the particle velocity at plane $\mathrm{A}$ in the upstream direction of the annular cavity at plane A can be expressed as

$$
u\left(l_{a}, r\right)=\frac{p_{p}\left(l_{a}, r\right)}{Z_{a}\left(l_{a}\right)}
$$


where $Z_{a}\left(l_{a}\right)$ is the normal impedance seen by the wave in region 3 (see Fig. 2) at plane A, i.e. at a distance $l_{a}$ from the left-end plate, in the axial direction. Then, the volume velocity is given by

$$
v_{l_{a}}=2 \pi \int_{r_{1}}^{r_{2}} u\left(l_{a}, r\right) r \mathrm{~d} r=\frac{2 \pi}{Z_{a}\left(l_{a}\right)} \int_{r_{1}}^{r_{2}} p_{p}\left(l_{a}, r\right) r \mathrm{~d} r
$$

where $p_{p}\left(l_{a}, r\right)$ and $u\left(l_{a}, r\right)$ are the pressure and particle velocity at a distance $l_{a}$ from the left endplate as a function of radial distance $r$ as shown in Fig. 2. Considering the continuity of the volume velocity at plane A, one gets

$$
\frac{S_{1}}{\rho_{0} c_{0}}\left(P_{1_{+}}-P_{1_{-}}\right)=\int_{S_{2}}\left(u_{2_{+}}-u_{2_{-}}\right) d S+\int_{S_{3}}\left(\frac{P_{p_{+}} \psi_{3 p_{+}}}{Z_{a}}-\frac{P_{p_{-}} \psi_{3 p_{-}}}{Z_{a}}\right) d S .
$$

One can divide the right-hand side of the above equation into two parts, viz. volume velocity due to the forward moving wave and that due to the rearward moving wave. So,

$$
\frac{S_{1}}{\rho_{0} c_{0}}\left(P_{1_{+}}-P_{1_{-}}\right)=\text {volume }_{+}-\text {volume }_{-},
$$

where

$$
\text { volume }_{+}=P_{p_{+}}\left[\int_{0}^{r_{1}}\left(\alpha_{2_{+}} \psi_{2 p_{+}}+\frac{1}{Z_{a}} \psi_{3 p_{+}}\right) 2 \pi r \mathrm{~d} r\right]
$$

and

$$
\text { volume }_{-}=P_{p_{-}}\left[\int_{0}^{r_{1}}\left(\alpha_{2_{-}} \psi_{2 p_{-}}+\frac{1}{Z_{a}} \psi_{3 p_{-}}\right) 2 \pi r \mathrm{~d} r\right] .
$$

So, in terms of the acoustic variables one can write the continuity equation of the volume velocity at plane $\mathrm{A}$ as

$$
v_{1}=P_{p_{+}}\left\{\alpha_{2_{+}} S_{2_{+}}+\frac{1}{Z_{a}} S_{3_{+}}\right\}-P_{p_{-}}\left\{\alpha_{2_{-}} S_{2_{-}}+\frac{1}{Z_{a}} S_{3_{-}}\right\} .
$$

Similarly, considering the continuity of volume velocity at plane B, one gets

$$
\begin{aligned}
v_{4}= & P_{p_{+}}\left\{\alpha_{2_{+}} S_{2_{+}}-\frac{1}{Z_{b}} S_{3_{+}}\right\} \mathrm{e}^{\left(-\mathrm{j} k_{z_{+}} l_{p}\right)} \\
& -P_{p_{-}}\left\{\alpha_{2_{-}} S_{2_{-}}-\frac{1}{Z_{b}} S_{3_{-}}\right\} \mathrm{e}^{\left(+\mathrm{j} k_{z_{-}} l_{p}\right)} .
\end{aligned}
$$

In the above equations, $S_{2_{ \pm}}$are given by Eq. (35) and $S_{3_{ \pm}}$are given as

$$
S_{3_{+}}=2 \pi \int_{r_{1}}^{r_{2}} \psi_{3 p_{+}}(r) r \mathrm{~d} r, \quad S_{3_{-}}=2 \pi \int_{r_{1}}^{r_{2}} \psi_{3 p_{-}}(r) r \mathrm{~d} r .
$$

Now, from Eqs. (36) and (51), one can write the state variables in a compact form as

$$
\left\{\begin{array}{c}
p_{1} \\
v_{1}
\end{array}\right\}=[\mathbf{A}]\left\{\begin{array}{l}
P_{p_{+}} \\
P_{p_{-}}
\end{array}\right\}
$$


where

$$
[\mathbf{A}]=\left[\begin{array}{cc}
\left(\frac{S_{2_{+}}}{S_{1}}\right) & \left(\frac{S_{2_{-}}}{S_{1}}\right) \\
\left(\alpha_{2_{+}} S_{2_{+}}+\frac{1}{Z_{a}} S_{3_{+}}\right) & -\left(\alpha_{2-} S_{2_{-}}+\frac{1}{Z_{a}} S_{3_{-}}\right)
\end{array}\right] .
$$

Similarly, one can relate the state variables at the entrance of region 4 to the pressure amplitudes of the forward and rearward waves in region 2. From Eqs. (37) and (52), one gets

$$
\left\{\begin{array}{c}
p_{4} \\
v_{4}
\end{array}\right\}=[\mathbf{B}]\left\{\begin{array}{l}
P_{p_{+}} \\
P_{p_{-}}
\end{array}\right\}
$$

where

$$
[\mathbf{B}]=\left[\begin{array}{cc}
\left(\frac{S_{2_{+}}}{S_{4}}\right) \mathrm{e}^{-\mathrm{j} k_{z_{+}} l_{p}} & \left(\frac{S_{2_{-}}}{S_{4}}\right) \mathrm{e}^{+\mathrm{j} k_{z_{-}} l_{p}} \\
\left(\alpha_{2_{+}} S_{2_{+}}-\frac{1}{Z_{b}} S_{3_{+}}\right) \mathrm{e}^{-\mathrm{j} k_{z_{+}} l_{p}} & -\left(\alpha_{2_{-}} S_{2_{-}}-\frac{1}{Z_{b}} S_{3_{-}}\right) \mathrm{e}^{+\mathrm{j} k_{z_{-}} l_{p}}
\end{array}\right]
$$

Now, one can eliminate the pressure amplitudes $P_{p_{ \pm}}$from Eqs. (54) and (56) to relate the acoustic variables at the up-stream and the down-stream points $u$ and $d$ (refer Fig. 2) as

$$
\left\{\begin{array}{c}
p_{1} \\
v_{1}
\end{array}\right\}=[\mathbf{T M}]\left\{\begin{array}{c}
p_{4} \\
v_{4}
\end{array}\right\}
$$

where

$$
[\mathbf{T M}]=[\mathbf{A}][\mathbf{B}]^{-1} .
$$

This transfer matrix between the up- and down-stream points of the perforated portion can be combined with transfer matrices of the up- and down-stream uniform pipes to get the overall transfer matrix. The transmission loss can then be calculated from the four pole parameters of the product transfer matrix [1].

At this point, it is of utmost interest to analyze the effect of the annular side cavities on the two ends of the perforated tube. When these lengths become zero, the impedances $Z_{a}$ and $Z_{b}$ tend to infinity. So matrices $\mathbf{A}$ and $\mathbf{B}$ take the form

$$
[\mathbf{A}]=\left[\begin{array}{cc}
\left(\frac{S_{2_{+}}}{S_{1}}\right) & \left(\frac{S_{2_{-}}}{S_{1}}\right) \\
\left(\alpha_{2_{+}} S_{2_{+}}\right) & -\left(\alpha_{2_{-}} S_{2_{-}}\right)
\end{array}\right]
$$

and

$$
[\mathbf{B}]=\left[\begin{array}{cc}
\left(\frac{S_{2_{+}}}{S_{4}}\right) \mathrm{e}^{-\mathrm{j} k_{z_{+}} l_{p}} & \left(\frac{S_{2_{-}}}{S_{4}}\right) \mathrm{e}^{+\mathrm{j} k_{z_{-}} l_{p}} \\
\left(\alpha_{2_{+}} S_{2_{+}}\right) \mathrm{e}^{-\mathrm{j} k_{z_{+}} l_{p}} & -\left(\alpha_{2_{-}} S_{2_{-}}\right) \mathrm{e}^{+\mathrm{j} k_{z_{-}} l_{p}}
\end{array}\right]
$$


Now making use of Eq. (59) and assuming $S_{1}$ to be equal to $S_{4}$, the final transfer matrix for a simple lined duct becomes

$$
[\mathbf{T M}]=\left[\begin{array}{cc}
\frac{\alpha_{2_{-}} \mathrm{e}^{\left(+\mathrm{j} k_{z_{+}} l_{p}\right)}+\alpha_{2_{+}} \mathrm{e}^{\left(-\mathrm{j} k_{z_{-}} l_{p}\right)}}{\alpha_{2_{-}}+\alpha_{2_{+}}} & \frac{\mathrm{e}^{\left(+\mathrm{j} k_{z_{+}} l_{p}\right)}-\mathrm{e}^{\left(-\mathrm{j} k_{z_{-}} l_{p}\right)}}{S_{1}\left(\alpha_{2_{-}}+\alpha_{2_{+}}\right)} \\
\frac{\alpha_{2_{-}} \alpha_{2_{+}} S_{1}\left(\mathrm{e}^{\left(+\mathrm{j} k_{z_{+}} l_{p}\right)}-\mathrm{e}^{\left(-\mathrm{j} k_{z_{-}} l_{p}\right)}\right)}{\left(\alpha_{2_{-}}+\alpha_{2_{+}}\right)} & \frac{\alpha_{2_{-}} \mathrm{e}^{\left(+\mathrm{j} k_{z_{+}} l_{p}\right)}+\alpha_{2_{+}} \mathrm{e}^{\left(-\mathrm{j} k_{z_{-}} l_{p}\right)}}{\alpha_{2_{-}}+\alpha_{2_{+}}}
\end{array}\right] .
$$

From this expression, it is seen that the four-pole parameters are independent of $S_{2_{+}}$and $S_{2_{-}}$if the unperforated lengths $l_{a}$ and $l_{b}$ tend to zero. One more very important point can be observed from Eq. (62). The term $\alpha_{2_{ \pm}}$corresponds to the term $Y^{ \pm}$in Eq. (6.7) of Ref. [1]. In that case, Eq. (62) boils down to Eq. (6.16) of Ref. [1] exactly. That shows the equivalence of the first and the third approach in the absence of any extended inlet or outlet portion in the protective layer. It is to be noted that in the derivation of Eq. (6.16) of Ref. [1], a similar left-moving wave with the same wavenumber (in the absence of mean flow) as the right-moving wave is assumed to be present in the duct. In the present paper, the third approach is similar to the first approach as far as the derivation of the axial wavenumber is concerned; the boundary conditions do not play a role here. So, both the first and the third approach do not take into account the precise effects of reflections at the boundaries as is implicit in the bulk reacting model. When extended inlet/outlet are not present in the third approach, the reflected wave at the end plate seems to be playing the same role as the assumed left-moving wave in the derivation of Eq. (6.16) of Ref. [1], thereby making the two approaches identical in this respect. When the extended inlet/outlet ducts are present, reflection in the annular cavities introduce some phase cancellation and modify the waves thereby making the first and the third approach different. So, in the absence of extended inlet/outlet the first approach can be used and the complicated process of integration at the boundaries is not necessary in these situations. These facts and effects of the non-zero values of these unperforated lengths $l_{a}$ and $l_{b}$ will be demonstrated with some sample mufflers in the subsequent sections.

\section{Validation of the third approach}

Although this approach is reasonably stable for analyzing mufflers of very large transverse dimensions as well, it is verified hereunder for some smaller mufflers as experimental results are not available for larger mufflers. The results from these methods are compared with the experimental results of Cummings and Chang [9,10] and those of Kirby [12] in Figs. 3 and 4.

The muffler dimensions taken for Fig. 3 are

$$
\begin{gathered}
r_{1}=1.98 \mathrm{~cm}, \quad r_{2}=3.80 \mathrm{~cm}, \\
l_{p}=32.5 \mathrm{~cm}, \quad l_{a}=0.00 \mathrm{~cm}, \quad l_{b}=0.00 \mathrm{~cm} .
\end{gathered}
$$




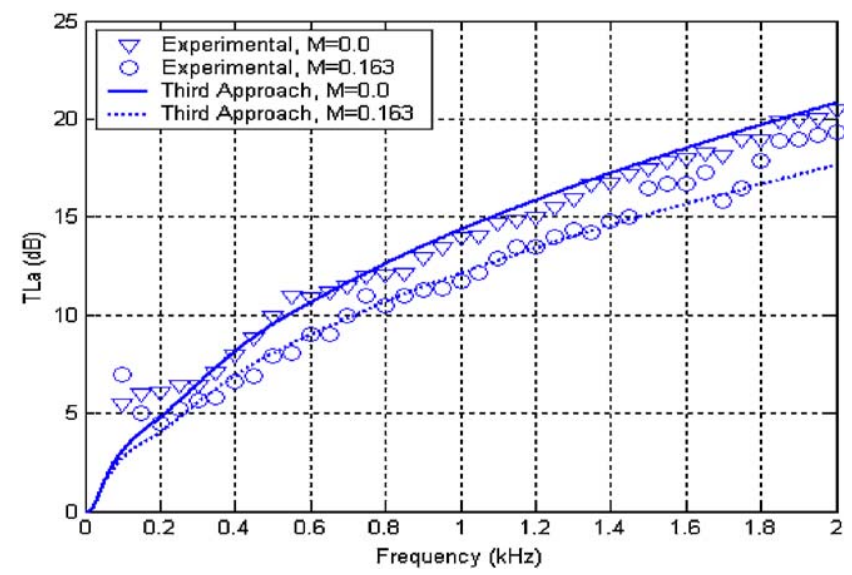

Fig. 3. Comparison of the third approach with the experimental results of Cummings and Chang [9].

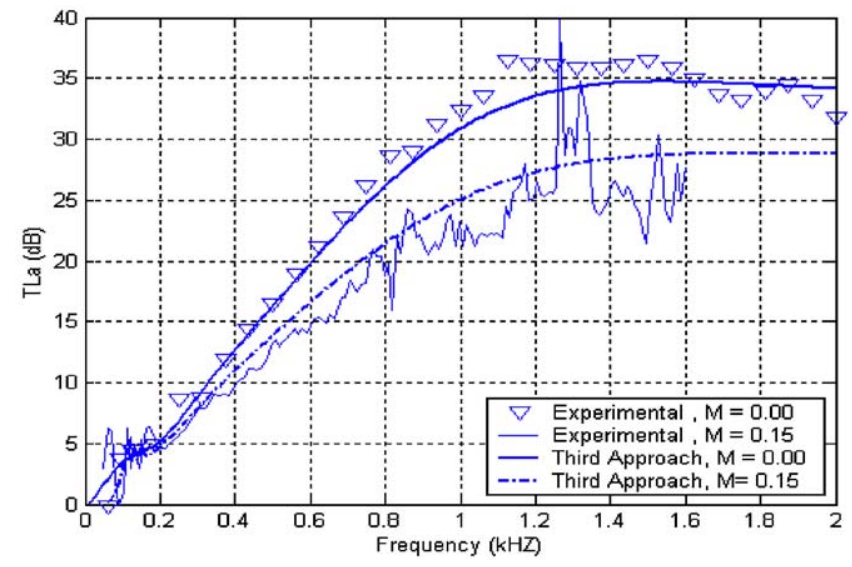

Fig. 4. Comparison of the third approach with the experimental results of Kirby [12].

These dimensions are assigned the same values as considered by Cummings and Chang [9] for their experiment and specified in the work of Peat and Rathi [11] who have used a finite element method to show that the experimental results obtained by Cummings and Chang are in good agreement with the numerical method used by them [11]. The flow resistivity of the material has been taken as $5000 \mathrm{Pas} / \mathrm{m}^{2}$. The complex wavenumber and the characteristic impedance of the material have been calculated using the curve-fitting formulas used by Cummings and Chang [9]. The perforated wall has not been considered as is the case in the reference. The deviation of the analytical results from the experimental ones at the low frequency zones are not very surprising as at these frequencies it is very difficult to get very accurate measurements. Moreover, the unperforated portions at the upstream end and at the downstream end (i.e. the extended inlet/ outlet) may play some role at these low frequencies. For all practical purposes, non-zero values of $l_{a}$ and $l_{b}$ are inevitable due to fabrication constraints. For all other frequency bands of interest, 
the model predictions are in very good agreement with the numerical results as well as experimental observations.

For a second test case, the model of the third approach is compared with the experimental results obtained by Kirby [12] for a relatively larger silencer with $r_{1}=3.8 \mathrm{~cm}, r_{2}=7.6 \mathrm{~cm}, l_{p}=$ $31.5 \mathrm{~cm}, l_{a}=l_{b}=0.0 \mathrm{~cm}$. The flow resistivity of the material has been taken as $30,700 \mathrm{~Pa} \mathrm{~s} / \mathrm{m}^{2}$ and the perforate porosity value has been taken as $26.3 \%$ as was considered by Kirby [12]. The bulk acoustic properties, $k_{w}$ and $Y_{w}$, are also calculated form the Delany and Bazley coefficients given by Kirby [12] using the empirical formula presented in the reference. The perforate impedance $Z_{p}$ has been calculated using Eq. (14). The predictions are again quite close to the experimental observations in nearly the entire frequency range of interest. Kirby, in his analysis, has neglected some higher order terms that are not so significant at low frequencies. But, their effects in the high-frequency region may be significant. After neglecting higher order terms from the polynomial expressions of the cylindrical functions, again some terms are neglected to put the final approximated eigenequation as a quadratic in $k_{x}^{2}$ for no flow condition, i.e. for $M=0$. Neglecting these terms may also lead to some inaccuracies in the high-frequency zones. In this paper, these approximated form or truncated series are assumed only for the first frequency step to calculate the initial guess to be used in the Newton-Raphson scheme. For subsequent steps, the root of the previous frequency step is used as the initial guess. That gives a better prediction of transmission loss at the higher frequency range as well as for larger silencer (of the order of $1 \mathrm{~m}$ diameter) to be discussed in subsequent sections.

\section{Comparison of the above three approaches}

Now that the third approach is verified to be predicting the transmission loss correctly over the frequency range of interest, the other two (approximate ones) are weighed against it to establish a standard procedure to exploit their advantages under the limitations they possess. For all the comparisons in this section, the muffler dimensions are same as those used for Fig. 3.

1. When $l_{a}$ and $l_{b}$ are not taken into consideration, i.e. on putting $l_{a}=l_{b}=0$, it is observed that the first and the third approach give exactly the same result (as shown above theoretically). The curves indistinguishably overlap each other as shown in Fig. 5.

2. When $l_{a}$ and $l_{b}$ are not zero, the first approach cannot be used directly as it does not take into account the extended inlet/outlet conditions while taking the slice from the infinite duct. However, a finite length of slice can be used in combination with an area change having extended inlet/outlet with side end walls being acoustically lined to approximately simulate the third approach. But, this shows considerable discrepancies with the third approach as can be seen from Fig. 6.

3. It can also be noted that the upward trends in the experimental results by Cummings and Chang in Fig. 3 in the high-frequency regions could be attributed to a deficiency or inadequacy in the heuristic derivation of the perforate impedance expression, i.e. Eq. (14).

4. The extended inlet/outlet on the two ends of the protective layer, however, can be implemented through the second approach. The comparison between this and the third approach is shown in Fig. 7. On comparing this with Fig. 3 it can be clearly observed that the second approach 


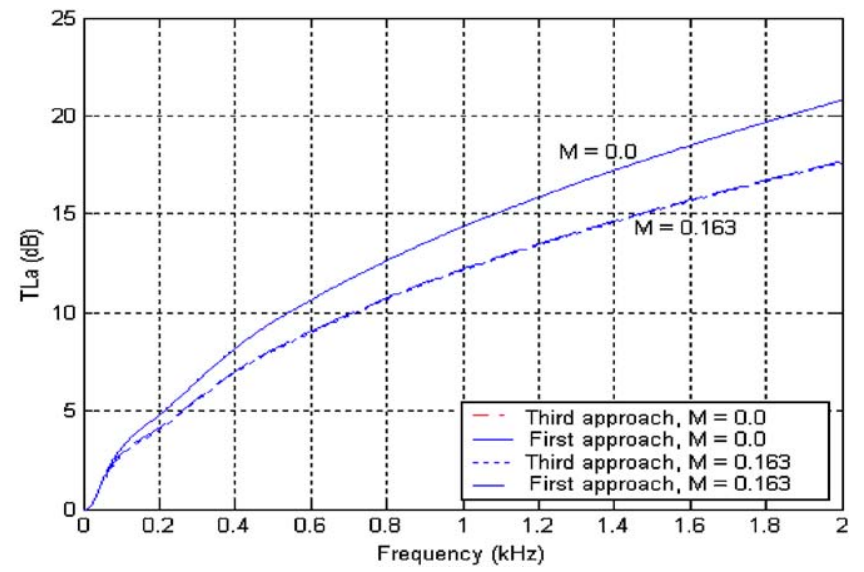

Fig. 5. Comparison of the first and the third approach. No extended inlet or outlet on the protective plate, i.e. $l_{a}=l_{b}=0.0$.

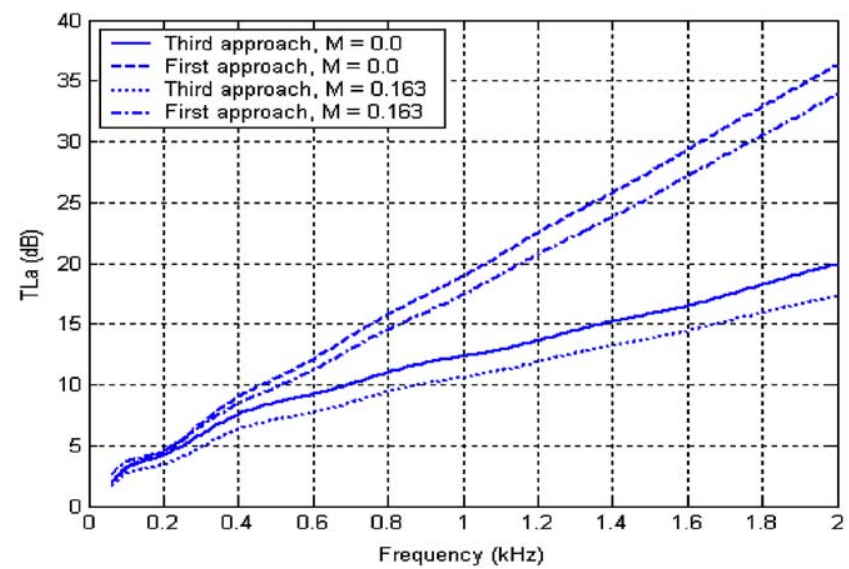

Fig. 6. Comparison of the first and the third approach. With extended inlet or outlet on the two ends of the protective plate, $l_{a}=2 \mathrm{~cm}$ and $l_{b}=3 \mathrm{~cm}$.

predictions differ considerably from the third approach and hence from the experimental observations.

\section{Some parametric studies making use of the numerical scheme}

The third approach can be used for large sized silencers too. It is used here to analyze two large mufflers and the results are shown in Fig. 8 for comparison. For the same absorptive material (flow resistivity $=10,000 \mathrm{~Pa} \mathrm{~s} / \mathrm{m}^{2}$ ) and same total length of $1 \mathrm{~m}$, the performance of very large mufflers is restricted to a very narrow range of frequency. It can be seen from Fig. 8 that the 


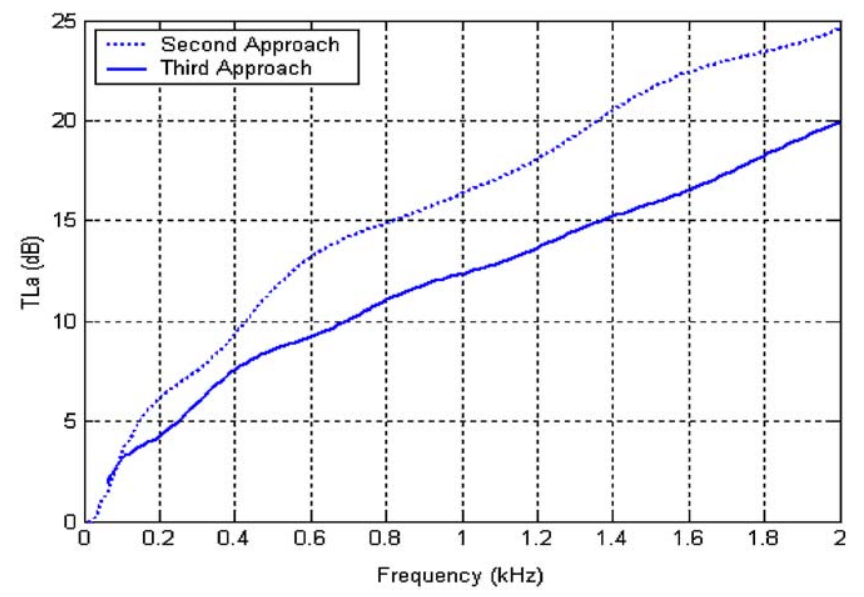

Fig. 7. Comparison of the second and the third approach for $M=0.0$. With extended inlet or outlet on the two ends of the protective plate, $l_{a}=2 \mathrm{~cm}$ and $l_{b}=3 \mathrm{~cm}$.

performance (the peak transmission loss) decreases with the increase in the size of the muffler if the thickness of the lining is kept constant which is not surprising. In the case of these mufflers (diameters of the order of $1 \mathrm{~m}$ ), a slight amount of instability is observed at high frequencies, as may be observed from Fig. 8.

The contribution of the reflection in the annular cavities $\left(L_{a}\right.$ and $\left.L_{b}\right)$ are seen in Fig. 9 to be very insignificant in the case of these large mufflers. So, for such large mufflers, small un-perforated lengths do not alter the performance greatly. Hence, in all the subsequent figures, these effects are not shown.

The relative thickness of the lining with respect to the diameter of the central air-way is generally expressed as the ratio $d / h, d$ being the lining thickness and $h$ the ratio of the crosssectional area to the wetted perimeter, which comes out to be half of $r_{1}$. Axial transmission loss $\left(T L_{a}\right)$ depends very strongly on this ratio as is implicit in Fig. 8; for curves (a) and (c), $d / h=1$ and $l / h=10$, and for curves (b) and (d), $d / h=0.5$ and $l / h=5$. The effect of lower value of $d / h$ is to narrow down the peak and lower value of $l / h$ is to lower the whole curve. The effect of $d / h$ alone is shown in Figs. 10 and 11.

First, the relative performance ( $T L_{a}$ curves) of mufflers for different values of $d / h$ ratio have been plotted keeping the radius of the central duct, $r_{1}$, constant. From Fig. 10, it can be observed that, at low values of $d / h$, the nature of the $T L_{a}$ curve is a narrow band. As the ratio is increased, the bandwidth of the $T L_{a}$ curve is increased, though a decrease in the $T L$ peak is also observed.

On the other hand, when outer radius, $r_{2}$, is held fixed, the effect of increasing $d / h$ is shown in Fig. 11. As $d / h$ increases, thickness of the lining gets increased, thereby making the $T L$ curve more and more flat over a larger band of frequency.

As the cross-sectional area of the central duct gets reduced, the mean flow velocity increases and therefore pressure drop will increase. So on the basis of these trends, it can be said that, though, increasing $d / h$ seems to be advantageous at first sight, a balance has to be maintained between increasing the outer radius and decreasing the inside radius. Incidentally, this is why pod silencers recommend themselves over simple lined ducts [8]. 


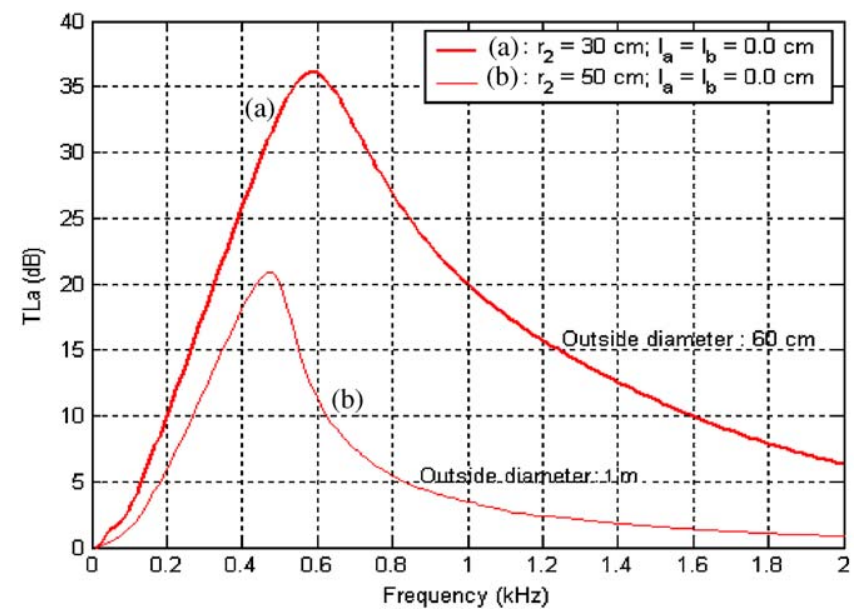

Fig. 8. Transmission loss curves for two large sized mufflers. (a) outside diameter $=60 \mathrm{~cm}$, inside diameter $=40 \mathrm{~cm}$; (b) outside diameter $=100 \mathrm{~cm}$, inside diameter $=80 \mathrm{~cm}$ as shown in the inset.

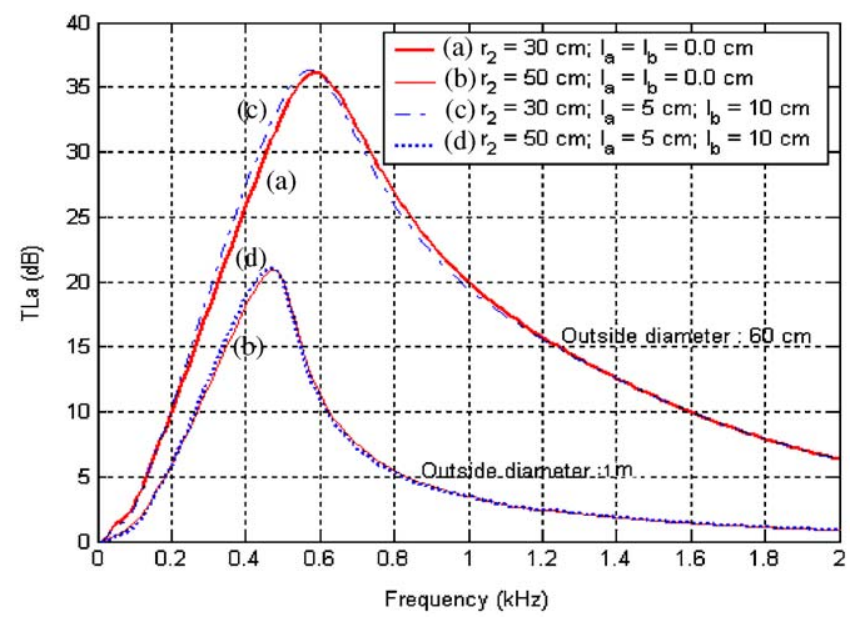

Fig. 9. Effect of annular cavities $\left(L_{a}\right.$ and $\left.L_{b}\right)$ for two large sized mufflers. (a) and (c) Outside diameter $=60 \mathrm{~cm}$, inside diameter $=40 \mathrm{~cm}$. (b) and (d) Outside diameter $=100 \mathrm{~cm}$, inside diameter $=80 \mathrm{~cm}$. (a) and (b) Without any unperforated lengths. (c) and (d) With unperforated lengths as shown in the inset.

\section{Concluding remarks}

When a lined duct with extended inlet and/or outlet is to be analyzed, the first approach fails completely to provide good predictions in line with the experimentally observed results. Though, the second approach is capable of analyzing such situations, it predicts somewhat higher values than the observed ones thereby leaving only the third approach to be used in such cases. 


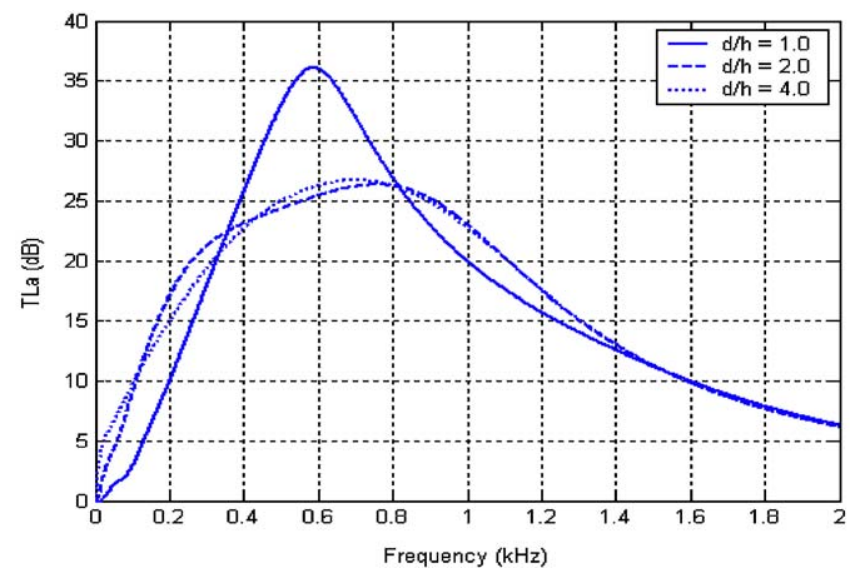

Fig. 10. Transmission loss curves for different $d / h$ values. Radius of the central duct, $r_{1}$ is held fixed at $20 \mathrm{~cm}$. Outer radius, $r_{2}$, only is varied. Length of the muffler $=1 \mathrm{~m}$; Flow resistivity $=10,000 \mathrm{~Pa} \mathrm{~s} / \mathrm{m}^{2} ; l_{a}=l_{b}=0.0$.

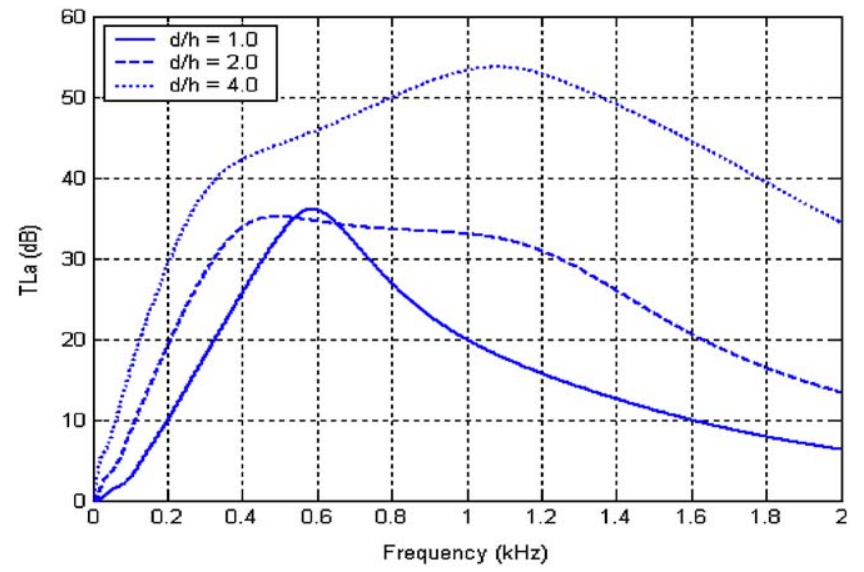

Fig. 11. Transmission loss curves for different $d / h$ values. Overall radius of the muffler, $r_{2}$ is held fixed at $30 \mathrm{~cm}$. Radius of the central duct, $r_{1}$, only is varied. Length of the muffler $=1 \mathrm{~m}$; Flow resistivity $=10,000 \mathrm{~Pa} \mathrm{~s} / \mathrm{m}^{2} ; l_{a}=l_{b}=0.0$.

For very large mufflers, the maximum difference in the axial transmission loss, with and without extended inlet/outlet, is below $1.0 \mathrm{~dB}$ as can be seen from Fig. 8. Hence, the effect of small extended inlet/outlets is not so significant in these large mufflers, in which case the first approach, which comes very handy for implementation, is sufficient to predict within tolerable limits.

In the absence of extended inlet/outlet, it has been shown that the first approach and the third approach are identically equal. So, in this case it is not worth performing the complicated process of imposing the boundary condition of the end plates.

While analyzing very large mufflers, the second method fails due to high numerical instabilities, which is not encountered in the first or the third approach. So, for such situations, the first approach is preferable owing to its simplicity. However, the third approach can be used to analyze 
the larger mufflers, if the extended inlet/outlet lengths are substantial portions of the overall length of the muffler.

Incidentally, the second approach would fail in the absence of the perforated plate which is required to sustain a pressure differential between the central flow duct and the absorptive annular duct. Therefore, this approach is basically not applicable to lined ducts.

\section{Acknowledgements}

Authors gratefully acknowledge the resources provided by the Department of Science and Technology of the Government of India through their Facility for Research in Technical Acoustics (FRITA). They are also thankful to Mr. Trinath Kar for his useful suggestions in some computational work. Authors are grateful to the reviewers for their patient reading, thoughtful comments and for their many suggestions for improving the manuscript.

\section{References}

[1] M.L. Munjal, Acoustics of Ducts and Mufflers, Wiley-Interscience, New York, 1987.

[2] A. Selamet, I.J. Lee, Z.L. Ji, N.T. Huff, Acoustic attenuation performance of perforated absorbing silencers, SAE Noise and Vibration Conference, Paper 2001-01-1435, 2001.

[3] M.L. Munjal, P.T. Thawani, Effect of protective layer on the performance of absorptive ducts, Noise Control Engineering Journal 45 (1) (1997) 14-18.

[4] K.S. Peat, A transfer matrix for an absorption silencer element, Journal of Sound and Vibration 146 (2) (1991) 353-360.

[5] M. Abramowitz, L.A. Stegun, Handbook of Mathematical Functions, Dover, New York, 1965.

[6] W. Frommhold, F.P. Mechel, Simplified method to calculate the attenuation of silencers, Journal of Sound and Vibration 141 (1) (1990) 103-125.

[7] F.P. Mechel (Ed.), Formulas of Acoustics, Springer, Berlin, 2002.

[8] M.L. Munjal, Analysis and design of pod silencers, Journal of Sound and Vibration 262 (3) (2003) $497-507$.

[9] A. Cummings, I.J. Chang, Sound attenuation of a finite length dissipative flow duct silencer with internal mean flow in the absorbent, Journal of Sound and Vibration 127 (1988) 1-17.

[10] A. Cummings, I.J. Chang, Internal mean flow effects on the characteristics of bulk-reacting liners in circular ducts, Acustica. 64 (4) (1987) 169-178.

[11] K.S. Peat, K.L. Rathi, A finite element analysis of the convected acoustic wave motion in dissipative silencers, Journal of Sound and Vibration 184 (3) (1995) 529-545.

[12] R. Kirby, Simplified techniques for predicting the transmission loss of a circular dissipative silencer, Journal of Sound and Vibration 243 (3) (2002) 403-426. 